\title{
An Exact Expression for Symbol-Error Rate of Multipulse PPM Technique in Free-Space Optics with Gamma-Gamma Channels
}

\author{
Ahmed E. Morra*, Haitham S. Khallaf ${ }^{\S}$, Hossam M. H. Shalaby ${ }^{\llbracket}$, and Zen Kawasakill \\ Egypt-Japan University of Science and Technology (E-JUST), Alexandria, Egypt \\ * Email: ahmed.morra@ejust.edu.eg, ahmedmorra@yahoo.com \\ § Email: haitham.khallaf@ejust.edu.eg, eng.h.khallaf@gmail.com \\ I Email: hossam.shalaby@ejust.edu.eg \\ || Email: kawasaki.zen@ejust.edu.eg
}

\begin{abstract}
Atmospheric turbulence, also called scintillation, is one of the major challenges that face free space optics (FSO) communications. In this paper, we derive both exact and approximate expressions for the average symbol-error rate (SER) of free-space optics systems adopting multipulse PPM (MPPM) technique in gamma-gamma channels. Our expressions are then verified by getting the same results using lognormal and exponential channel expressions where lognormal and exponential distributions are valid only for weak and strong turbulence, respectively. As the computation of the exact expression is time consuming, we get an approximate expression based on Gauss-Laguerre quadrature. Both expressions produce almost the same results. Finally, we make a comparison between the average SER performance of MPPM and PPM assuming equal average energy per bit and equal data rate. We found that the performance of MPPM is better than that of PPM in case of weak turbulence but at strong turbulence both MPPM and PPM have nearly the same performance.
\end{abstract}

\section{INTRODUCTION}

The need for very high data rate point-to-point communications can be fulfilled using a promising technology called freespace optics (FSO) [1]. FSO has several advantages over radiofrequency (RF) technology such as extremely high bandwidth, license-free, and interference immunity. Because of all of these advantages, FSO is used for a variety of applications such as last mile connectivity and optical-fiber backup [2]. However, since FSO uses atmosphere as transmission medium so its performance is affected by many challenges like atmospheric attenuation, scintillation, window attenuation, alignment or building motion, solar interference, and line-of-sight obstructions particularly over ranges greater than $1 \mathrm{~km}$ [1]. One important phenomenon that degrades the performance of FSO is atmospheric turbulence or scintillation. Scintillation results in random fluctuations in both the amplitude and the phase of the received signal which can degrade the FSO performance and leads to increasing in the bit error rate (BER) [1].

Both on-off keying (OOK) and PPM techniques have been widely used in FSO. In addition, multipulse PPM (MPPM) has been recently proposed as an alternative modulation technique to OOK and PPM because it is more bandwidth efficient than PPM, yet slightly more complex [1], [3]. In fact MPPM can be considered as a generalization to PPM, where $n \in\{1,2, \ldots, M / 2\}$ pulses are transmitted during a symbol frame of $M$ slots and as a result MPPM frame will transmit $\log _{2}\left(\begin{array}{c}M \\ n\end{array}\right)$ bits and this is the reason why MPPM is more bandwidth efficient than PPM.

The studies that have been made to investigate the performance of MPPM in the case of FSO in gamma-gamma channels are rare. First Hamkins and Moision have obtained an exact expression for the symbol-error rate (SER) of MPPM in the case of discrete memoryless channels in non turbulent atmosphere [4]. Nguyen and Lampe have studied coded MPPM FSO transmission using discrete-time Poisson channel model in non turbulent atmosphere. They have studied two issues, first when MPPM is better than PPM and second how MPPM could be used with error control coding [5]. Gappmair and Muhammad have got an exact expression for the SER of PPM under gamma-gamma optical scintillation model [6]. Xu et. al have discussed the use of binary convolutional coding with iterative detection for the case of MPPM modulation [1], [7]. Wilson et. al have studied the use of multiple-input/multipleoutput (MIMO) channel for FSO adopting MPPM. They have concluded that the resulting MIMO channel can reduce FSO turbulent effects in both log-normal and Rayleigh-fading channel models [8]. Balsells et al. have calculated the average BER for a rate adaptive transmission technique using MPPM block coding of variable Hamming weight under turbulence conditions [9]. This type of block coding has a variable amount of pulses and has been shown to have a high peak to average optical power ratio. Their analysis was based on a hyperexponential fitting and Monte Carlo simulation.

Up till now, no one has got an exact expression for the average SER of MPPM in the case of FSO under gammagamma distribution. In this paper, we derive both exact and approximate (based on Gauss-Laguerre quadrature) expressions for the average SER of this system and verify our mathematical expressions by getting the same results using lognormal and exponential channel expressions where lognormal and exponential distributions are valid only for weak and strong turbulence, respectively [10], [11]. In fact, gamma- 
gamma distribution has been proposed as a general scheme as it is valid for both weak and strong turbulence [11]. Finally, we make comparison between the average SER performance of both MPPM and PPM assuming equal average energy per bit and equal data rate.

\section{MPPM AVERAGE SER ANALYSIS IN ATMOSPHERIC TURBULENCE CHANNEL}

\section{A. MPPM SER in Non Turbulent Atmosphere}

At the receiver side, we assume that the received photon count per MPPM slot follows a Poisson distribution. In that case the SER is given by [4] after slight modifications:

$$
\begin{aligned}
& P_{e}=\sum_{K_{m i n}=0}^{\infty} \sum_{l=1}^{M-n} \sum_{m=1}^{n}\left(\begin{array}{c}
n \\
m
\end{array}\right)\left(\begin{array}{c}
M-n \\
l
\end{array}\right) p_{1}(K \text { min })^{m} \\
& \times\left(1-P_{1}\left(K_{\text {min }}\right)\right)^{n-m}\left[P_{0}\left(K_{\text {min }}\right)^{M-n-l}\left(1-P_{0}\left(K_{\text {min }}\right)\right)^{l}\right. \\
& \left.+p_{0}(K \text { min })^{l} P_{0}(K \min -1)^{M-n-l}\left(1-\frac{1}{\left(\begin{array}{c}
l+m \\
m
\end{array}\right)}\right)\right]
\end{aligned}
$$

where $M$ is the number of slots per frame, $n$ is the number of signal slots, $K_{\min }$ is the minimum photon count in symbol signal slots, and

$$
\begin{gathered}
p_{0}\left(K_{\text {min }}\right)=\frac{K_{b}^{K_{\text {min }}}}{K_{\text {min }} !} e^{-K_{b}} \\
p_{1}\left(K_{\text {min }}\right)=\frac{\left(K_{s}+K_{b}\right)^{K_{\text {min }}}}{K_{\text {min }} !} e^{-\left(K_{s}+K_{b}\right)} \\
P_{1}\left(K_{\text {min }}\right)=\sum_{j=0}^{K m i n} \frac{\left(K_{s}+K_{b}\right)^{j}}{j !} e^{-\left(K_{s}+K_{b}\right)} \\
P_{0}\left(K_{\text {min }}\right)=\sum_{j=0}^{K m i n} \frac{K_{b}^{j}}{j !} e^{-K_{b}}
\end{gathered}
$$

Here, $K_{b}$ and $K_{b}+K_{s}$ denote the mean values of photon count in non-signal and signal slots, respectively.

\section{B. Channel Models}

Several channel models have been assumed to model atmospheric turbulence in literature, however, the most commonly used statistical models are:

- Lognormal distribution: This is suitable in weak turbulence and its probability density function (PDF) is [10]:

$$
f\left(K_{s}\right)=\frac{1}{K_{s} \sqrt{2 \pi \sigma_{l}^{2}}} e^{-\frac{\left(\ln K_{s}-\mu_{l}\right)^{2}}{2 \sigma_{l}^{2}}}, K_{s} \geqslant 0
$$

where $\sigma_{l}^{2}=\ln \left(1+\chi_{S C}\right), \mu_{l}=\ln (\lambda)-\frac{1}{2} \sigma_{l}^{2}, \chi_{S C}=$ $E\left[K_{s}^{2}\right] / E\left[K_{s}\right]^{2}-1$ is the scintillation index, and $\lambda$ is the average received photon count (the average of $K_{s}$ ).

- Exponential distribution: This is suitable in strong turbulence and its PDF is [10]:

$$
f\left(K_{s}\right)=\frac{1}{\lambda} e^{-\frac{K_{s}}{\lambda}}, K_{s} \geqslant 0
$$

- Gamma-Gamma distribution: This is suitable in both strong and weak turbulence so we will use it in our analysis and its PDF is given by [11]:

$$
\begin{aligned}
f\left(K_{s}\right)= & \frac{2(\alpha \beta)^{\frac{(\alpha+\beta)}{2}}}{\lambda \Gamma(\alpha) \Gamma(\beta)}\left(\frac{K_{s}}{\lambda}\right)^{\frac{(\alpha+\beta)}{2}-1} \\
& \times K_{\alpha-\beta}\left(2 \sqrt{\frac{\alpha \beta K_{s}}{\lambda}}\right)
\end{aligned}
$$

where

$$
\begin{gathered}
\alpha=\left(\exp \left[\frac{0.49 \sigma_{R}^{2}}{\left(1+1.11 \sigma_{R}^{\frac{12}{5}}\right)^{\frac{7}{6}}}\right]-1\right)^{-1} \\
\beta=\left(\exp \left[\frac{0.51 \sigma_{R}^{2}}{\left(1+0.69 \sigma_{R}^{\frac{12}{5}}\right)^{\frac{5}{6}}}\right]-1\right)^{-1}
\end{gathered}
$$

where $\alpha$ and $\beta$ are the scintillation parameters that are dependent on $\sigma_{R}^{2}, \sigma_{R}^{2}$ is unitless Rytov variance, $\Gamma($.$) is the gamma function, and K_{c}($.$) denotes the c_{t h}$ order modified Bessel function of the second kind. The scintillation index is related to $\alpha$ and $\beta$ as

$$
\chi_{S C}=\frac{1}{\alpha}+\frac{1}{\beta}+\frac{1}{\alpha \beta} .
$$

\section{Exact Mathematical Analysis of MPPM Average SER with Gamma-Gamma Distribution}

In our analysis we assume that we work in a clear atmosphere conditions and also both the transmitter and receiver are fixed and perfectly aligned. Scintillation or channel fading, however, is considered in our FSO performance derivation. The average SER can be obtained by averaging $P_{e}$ with respect to $K_{s}$ using a gamma-gamma model. Because $K_{s}$ is the only parameter that depends on the channel distribution so $p_{1}\left(K_{\text {min }}\right)^{m}\left(1-P_{1}\left(K_{m i n}\right)\right)^{n-m}$ is the only term that will be averaged with respect to $K_{s}$. After rigorous mathematical analysis, we are able to show that the average SER for MPPM under gamma-gamma distribution is obtained by replacing $p_{1}\left(K_{\min }\right)^{m}\left(1-P_{1}\left(K_{\min }\right)\right)^{n-m}$ in (1) by the value of $P_{2}\left(K_{\min }\right)$ where

$$
\begin{aligned}
& P_{2}\left(K_{\text {min }}\right)= \\
& \int_{0}^{\infty} p_{1}\left(K_{\text {min }}\right)^{m}\left(1-P_{1}\left(K_{\text {min }}\right)\right)^{n-m} f\left(K_{s}\right) d K_{s} \\
= & \int_{0}^{\infty}\left[\frac{\left(K_{s}+K_{b}\right)^{K_{\text {min }}}}{K_{\min } !} e^{-\left(K_{s}+K_{b}\right)}\right]^{m} \\
\times & {\left[1-\sum_{j=0}^{K \min } \frac{\left(K_{s}+K_{b}\right)^{j}}{j !} e^{-\left(K_{s}+K_{b}\right)}\right]^{n-m} f\left(K_{s}\right) d K_{s} }
\end{aligned}
$$




$$
\begin{aligned}
= & \sum_{j_{1}=K_{m i n}+1}^{\infty} \sum_{j_{2}=K_{m i n}+1}^{\infty} \ldots \ldots \sum_{\substack{j_{n-m}=K_{m i n}+1 \\
j_{1}+j_{2}+\ldots .+j_{n-m}+m K_{m i n}}}^{\infty} \sum_{i=0}^{e^{-n K_{b}}} \\
& \frac{2\left(\frac{\alpha \beta}{\lambda}\right)^{\frac{\alpha+\beta}{2}}}{\Gamma(\alpha) \Gamma(\beta)}\left(j_{1}+j_{2}+\ldots . .+j_{n-m}+m K_{m i n}\right) \\
& \times K_{b}^{j_{1}+j_{2}+\ldots . .+j_{n-m}+m K_{m i n}-i} \int_{0}^{\infty} K_{s}^{i+\frac{\alpha+\beta}{2}-1} \\
& \times e^{-n K_{s}} K_{\alpha-\beta}\left(2 \sqrt{\frac{\alpha \beta K_{s}}{\lambda}}\right) d K_{s}
\end{aligned}
$$

Let

$$
p=\int_{0}^{\infty} K_{s}^{i+\frac{(\alpha+\beta)}{2}-1} e^{-n K_{s}} K_{\alpha-\beta}\left(2 \sqrt{\frac{\alpha \beta K_{s}}{\lambda}}\right) d K_{s}
$$

By using [12] we found that the value of $p$ will be,

$$
\begin{gathered}
p=\frac{\Gamma(i+\alpha) \Gamma(i+\beta)}{2 \sqrt{\frac{\alpha \beta}{\lambda}}} e^{\left(\frac{\alpha \beta}{2 n \lambda}\right)} n^{-\left(i+\frac{(\alpha+\beta)}{2}-\frac{1}{2}\right)} \\
\times W_{-\left(i+\frac{(\alpha+\beta)}{2}-\frac{1}{2}\right), \frac{1}{2}(\alpha-\beta)}\left(\frac{\alpha \beta}{n \lambda}\right)
\end{gathered}
$$

where $W_{\lambda, \mu}(z)$ is the Whittaker function. Then we are able to show that,

$P_{2}\left(K_{\text {min }}\right)=$

$$
\left\{\begin{array}{l}
\sum_{i=0}^{m K_{\min }} \frac{\left(\frac{\alpha \beta}{\lambda}\right)^{\frac{\alpha+\beta-1}{2}}}{\Gamma(\alpha) \Gamma(\beta)}\left(\begin{array}{c}
K_{\min } \\
i
\end{array}\right) e^{\left(-n K_{b}\right)} K_{b}^{m K_{\min }-i} \\
\times \frac{\Gamma(i+\alpha) \Gamma(i+\beta)}{K_{\min } ! m} e^{\left(\frac{\alpha \beta}{2 n \lambda}\right)} n^{-\left(i+\frac{(\alpha+\beta)}{2}-\frac{1}{2}\right)} \\
\times W_{-\left(i+\frac{(\alpha+\beta)}{2}-\frac{1}{2}\right), \frac{1}{2}(\alpha-\beta)}\left(\frac{\alpha \beta}{n \lambda}\right), \quad \text { for } n=m \\
j=(n-m)\left(K_{\min }+1\right) \\
\times\left(\begin{array}{l}
j+m K_{\min } \\
i
\end{array}\right) e^{\left(-n K_{b}\right)} \frac{K_{b}^{j+m K_{m i n}-i}}{\Gamma(\alpha) \Gamma(\beta)} \\
\times \frac{\Gamma(i+\alpha) \Gamma(i+\beta)}{K_{\min } ! m} f(j) e^{\left.\left(\frac{\alpha \beta}{2 n \lambda}\right) n^{-\left(i+\frac{(\alpha+\beta)}{2}\right.}-\frac{1}{2}\right)} \\
\times W_{-\left(i+\frac{(\alpha+\beta)}{2}-\frac{1}{2}\right), \frac{1}{2}(\alpha-\beta)}\left(\frac{\alpha \beta}{n \lambda}\right), \quad \text { for } n \neq m
\end{array}\right.
$$

To define $f(j)$, let we have a set of vectors, each has a dimension of $(n-m)$. The vector elements are integer and are between $\left(K_{\text {min }}+1\right)$ and $j-(n-m-1)\left(K_{\text {min }}+1\right)$. In addition, the summation of all elements of any vector is equal to $j$ :

$$
\begin{aligned}
& f(j)= \\
& \sum_{i=1}^{\text {no. of vectors }} \frac{1}{\prod_{s=1}^{n-m}(\text { element number } s \text { in vector number } i) !}
\end{aligned}
$$

D. Approximate Expression of MPPM Average SER with Gamma-Gamma Distribution

The computation complexity of the exact SER expression given in the last subsection is high and time consuming. This motivates us to obtain an approximate expression based on Gauss-Laguerre quadrature rule [13]. This method produces faster results with good approximation. Using this method, it is easy to show that

$$
\begin{aligned}
P_{2}\left(K_{\text {min }}\right)= & \sum_{i=1}^{c} w_{i} \frac{e^{-n K_{b}}}{K_{\min } !^{m}}\left(x_{i}+K_{b}\right)^{m K_{\min }} \\
& \times\left[e^{\left(x_{i}+K_{b}\right)}-\sum_{j=0}^{K_{\min }} \frac{\left(x_{i}+K_{b}\right)^{j}}{j !}\right]^{n-m} f\left(x_{i}\right)
\end{aligned}
$$

where $c>1$ denotes the number of terms; and for any $i \in$ $\{1,2, \ldots, c\}, x_{i}$ is the $i$ th root of Laguerre polynomial $L_{c}(x)$ with degree $c, w_{i}$ is the corresponding weighting coefficient [13], and $f\left(x_{i}\right)$ is the atmospheric turbulence distribution.

\section{NUMERICAL RESUlTS}

\section{A. Numerical Results}

In this subsection, we numerically evaluate both exact and approximate expressions of the symbol-error rates obtained in the previous subsections for different channel parameters. In our evaluation we use MPPM frames of size $M=8$ slots and $n=4$ pulses per frame. The average photon count for background noise is assumed to be $K_{b}=1$.

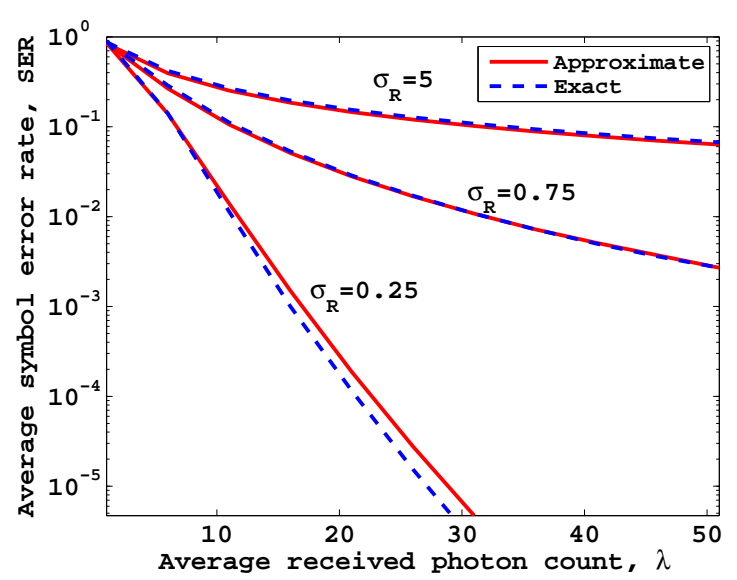

Fig. 1. Average symbol-error rate (SER) versus average received signal photon count $\lambda$ using both exact and approximate methods under gammagamma distribution with $M=8, n=4$, and $K_{b}=1$. 
In Fig. 1 we compare between the exact and approximate expressions under gamma-gamma atmospheric channel with weak, moderate, and strong turbulence conditions of $\sigma_{R}=$ $0.25,0.75$, and 5 , respectively. We notice from the figure that both exact and approximate expressions are nearly identical but the computation complexity is much reduced when using the latter.

Figure 2 illustrates the change of average SER with the average received photon count $\lambda$ under gamma-gamma channel. In our evaluations, we have used the approximate method with Laguerre polynomial degree $c=50$. We have studied weak, moderate, and strong turbulence conditions of $\sigma_{R}=$ $0.25,0.75$, and 5 , respectively. As shown in the figure, in case of the weak turbulence the system performance will be improved by increasing the average received photons because this will improve the signal to noise ratio (SNR). As the turbulence level increases turbulence will have the dominate effect on the system performance, so the performance will be degraded for both moderate and strong turbulence. The symbol-error rates of the MPPM system under both exponential and lognormal channels (which are valid only for strong and weak turbulence, respectively) are also included in the figure for the sake of comparison. The variance of the lognormal distribution is governed by the same scintillation index as that of the gammagamma model, which is equal to $0.062,0.475$, and 1.214 for weak, moderate and, strong turbulence respectively. As expected, at strong turbulence with $\sigma_{R}=5$, the gammagamma model approaches the exponential model. On the other hand, the gamma-gamma model approaches the lognormal one as $\sigma_{R}$ decreases. So at weak turbulence $\left(\sigma_{R}=0.25\right)$ the gamma-gamma model reflects more lognormal model than that at moderate turbulence $\left(\sigma_{R}=0.75\right)$.

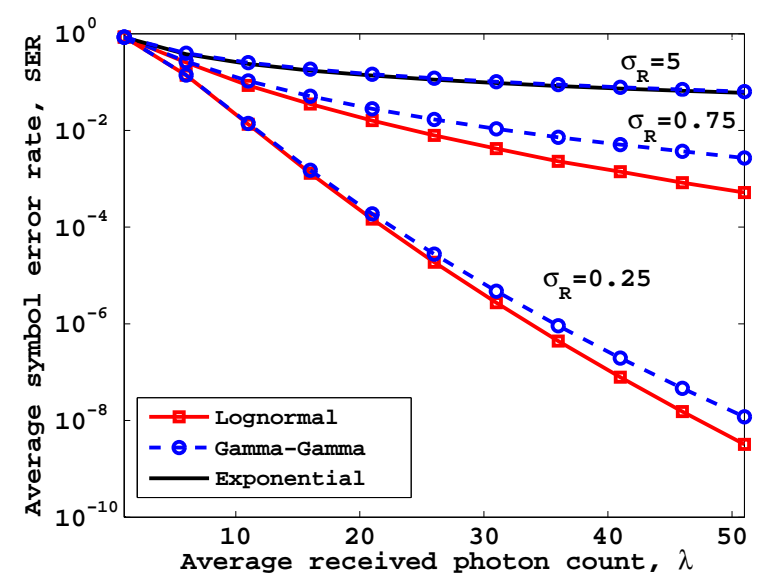

Fig. 2. Average SER versus average received signal photon count $\lambda$ using the approximate method for different channel models with $M=8, n=4$, and $K_{b}=1$.

\section{COMPARISON BETWEEN MPPM AND PPM AVERAGE SERS}

In this section we make a comparison between the performance of MPPM and PPM systems under different turbulence

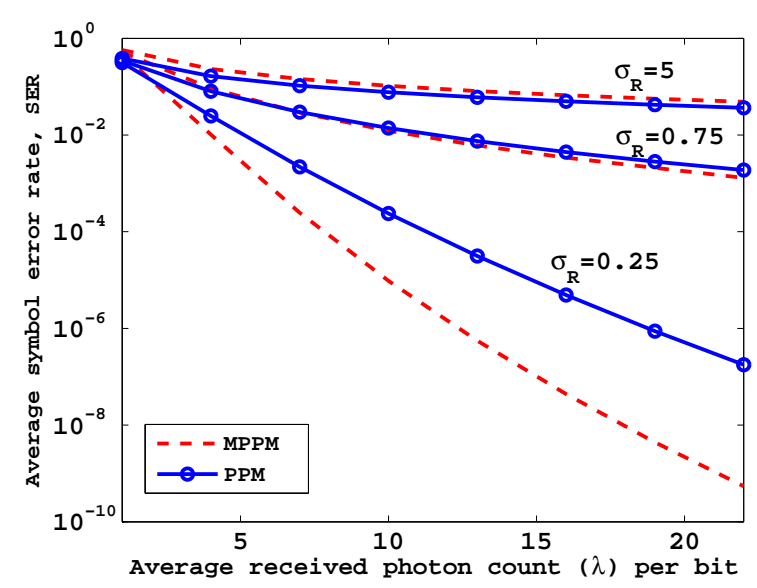

Fig. 3. Average SER versus average received photon count $(\lambda)$ per bit for MPPM with $M=11, n=2$, and PPM with $M=3$ with equal data rate.

conditions. In general in case of transmitting the same data rate using the same average energy per bit the performance of the MPPM will have high immunity to noise if it is compared to the PPM because MPPM will have higher peak power so higher signal to noise ratio. At weak turbulence, the noise has the dominant effect on the performance of the system so as shown in Fig. 3, the performance of MPPM is better than PPM in that case. However, at strong turbulence, the turbulence has the dominant effect on the performance of the system so as shown in Fig. 3, both MPPM and PPM have nearly the same performance.

\section{CONCLUSiON}

Both exact and approximate expressions for average SER of free-space optics systems adopting MPPM technique in gamma-gamma channels have been derived. Our expressions have been verified using both lognormal and exponential channel models. As the computation of the exact expression is time consuming, we get an approximate expression based on Gauss-Laguerre quadrature. Both expressions produce almost the same results. Finally, we have made a comparison between the average SER performances of both MPPM and PPM schemes, assuming both equal average energy per bit and equal data rate. We have found that the performance of MPPM is better than that of PPM in case of weak turbulence but at strong turbulence both MPPM and PPM have nearly the same performance.

\section{ACKNOWLEDGEMENT}

The authors would like to thank Egyptian Ministry of Higher Education (MoHE) and Egypt-Japan University of Science and Technology (E-JUST) for their support.

\section{REFERENCES}

[1] F. Xu, M. A. Khalighi, and S. Bourennane, "Coded PPM and multipulse PPM and iterative detection for free-space optical links," Journal of Optical Communications and Networking, vol. 1, no. 5, pp. 404-415, Oct. 2009. 
[2] X. Yi, Z. Liu, P. Yue, and T. Shang, "BER performance analysis for M-ary PPM over gamma-gamma atmospheric turbulence channels," in Proc. 6th Wireless Commun. Networking and Mobile Computing (WiCOM 2010), Sept. 2010.

[3] H. Sugiyama and K. Nosu, "MPPM: a method for improving the band utilization efficiency in optical PPM," J. Lightw. Technol., vol. 7, no. 3, pp. 465-472, Mar. 1989.

[4] J. Hamkins and B. Moision, "Multipulse pulse-position modulation on discrete memoryless channels," The Interplanetary Network Progress Report, vol. 42, no. 161, pp. 1-13, May 2005.

[5] T. Nguyen and L. Lampe, "Coded multipulse pulse-position modulation for free-space optical communications," IEEE Trans. Commun., vol. 58, no. 4, pp. 1036-1041, Apr. 2010.

[6] W. Gappmair and S. S. Muhammad, "Error performance of PPM/Poisson channels in turbulent atmosphere with gamma-gamma distribution," Electronics Letters, vol. 43, no. 16, pp. 2-3, Aug. 2007.

[7] F. Xu, M. Khalighi, and S. Bourennane, "Efficient channel coding for multipulse pulse position modulation in terrestrial FSO systems," Proc. of SPIE, vol. 7464, pp. 74640M-1-74640M-12, Aug. 2009.

[8] S. G. Wilson, M. B. Pearce, Q. Cao, and M. Baedke, "Optical repetition MIMO transmission with multipulse PPM," IEEE J. Sel. Areas Commun., vol. 23, no. 9, pp. 1901-1910, Sept. 2005.

[9] J. M. G. Balsells, A. J. Navas, J. F. Paris, M. C. Vazquez, and A. P. Notario, "Closed-form BER analysis of variable weight MPPM coding under gamma-gamma scintillation for atmospheric optical communications." Optics letters, vol. 37, no. 4, pp. 719-721, Feb. 2012

[10] K. Kiasaleh, "Performance of APD-based, PPM free-space optical communication systems in atmospheric turbulence," IEEE Trans. Commun., vol. 53, no. 9, pp. 1455-1461, Sept. 2005.

[11] R. L. Al-Habash, M. A. Andrews, and L. C. Phillips, "Mathematical model for the irradiance probability density function of a laser beam propagating through turbulent media," Opt. Eng., vol. 40, no. 8, pp. 1554-1562, Aug. 2001.

[12] I. I. M. Gradshteyn and Ryzhik, Table of Integrals, Series, and Products, 7th ed., A. Jeffrey and D. Zwillinger, Eds. Academic Press, Mar. 2007.

[13] M. Abramowitz and I. A. Stegun, Eds., Handbook of mathematical functions, 10th ed. Dover Publications, Dec. 1972 
This page is intentionally left blank. 